\title{
Fundamental Semigroups for Local Control Sets
}

\author{
Fritz Colonius* $^{*}$ Luiz A.B. San Martin ${ }^{\dagger} \quad$ Marco Spadini ${ }^{\ddagger}$
}

March 25, 2003

\begin{abstract}
In this paper we associate a semigroup to a locally maximal subset of complete controllability, i.e., a local control set. This fundamental semigroup is based on equivalence classes under homotopies in the set of trajectories. It reflects the structure of the set of closed (trajectory) loops in the local control set. We discuss the relations between different local control sets and prove a Van Kampen-type theorem for their unions and intersections.
\end{abstract}

\section{Introduction}

This paper studies controllability properties of nonlinear control systems described by ordinary differential equations. More precisely, we study topological properties of locally maximal subsets of complete controllability, i.e., local control sets as introduced in [4]. We associate a semigroup $\Lambda\left(D, p_{0}\right)$ to every pointed local control set $\left(D, p_{0}\right)$, i.e., to a local control set with one (inner) point $p_{0}$ singled out. This fundamental semigroup $\Lambda\left(D, p_{0}\right)$ is based on equivalence classes under homotopies in the set of trajectories. It

*Institut für Mathematik, Universität Augsburg, 86135 Augsburg, Germany, e-mail: colonius@math.uni-augsburg.de

†Departmento de Matématica. Universidade Estadual de Campinas. Cx. Postal 6065. 13083-859 Campinas - SP, Brasil. e-mail: smartin@ime.unicamp.br

‡Dipartimento di Matematica Applicata, Via S. Marta 3, 50139 Firenze, Italy, e-mail: spadini@dma.unifi.it 
is adapted to the dynamics of the system and reflects the structure of the set of closed (trajectory-) loops in $D$. It enables us to distinguish between different nested local control sets. Furthermore, we discuss the relations between different local control sets and prove a Seifert-Van Kampen-type theorem for the union and intersection of local control sets; compare, e.g., tom Dieck [13] or Massey [9] for this classical theorem for homotopy groups.

Homotopy properties of control sets in semisimple Lie groups have been discussed by San Martin and Santana in [10]. Sarychev discussed homotopy properties of the set of trajectories for completely controllable systems, and in particular, for systems without drift in [11], [12]. Lawson [8] considered homotopies in the context of semigroups in Lie groups. Preliminary results on our construction of the semigroup have appeared in [3].

In Section 2 we present some preliminary results on local control sets and, in particular, on the different parts of their boundaries. Section 3 specifies conditions which ensure that the intersections and unions of local control sets are again local control sets. In Section 4 we construct the fundamental semigroup and discuss some simple properties and examples. Finally, Section 5 presents the main result, a Seifert-Van Kampen-type theorem describing the fundamental semigroup of a union of two local control sets; here relative fundamental semigroups have to be used which are obtained by collapsing the intersection to a point. The proof is based on a careful discussion of the entrance and exit behavior of local control sets.

Notation 1.1 Besides the function space $L^{\infty}\left(\mathbb{R}, \mathbb{R}^{d}\right)$ with norm $\|\cdot\|_{\infty}$, we shall consider the Sobolev space $W^{1, \infty}\left(\mathbb{R}, \mathbb{R}^{d}\right)$ endowed with the norm $\|x\|_{W^{1, \infty}}=$ $\|x\|_{\infty}+\|\dot{x}\|_{\infty}$.

\section{Preliminaries}

In this section, we introduce the basic notions and formulate preliminary results on local control sets that we use throughout the paper.

Consider the system

$$
\dot{x}(t)=f(x(t), u(t)), \quad u \in \mathcal{U},
$$

where $\mathcal{U}$ denotes the set of all piecewise continuous functions taking values in the compact subset $U$ of $\mathbb{R}^{m}$, and $f: \mathbb{R}^{d} \times \mathbb{R}^{m} \rightarrow \mathbb{R}^{d}$ is $C^{1}$. We will endow $\mathcal{U}$ with the topology inherited by the inclusion $\mathcal{U} \subset L^{\infty}\left(\mathbb{R}, \mathbb{R}^{m}\right)$. We assume 
that unique solutions $\varphi\left(t, x_{0}, u\right), t \in \mathbb{R}$, exist for all considered $x_{0} \in \mathbb{R}^{d}$ and $u \in \mathcal{U}$.

System (1) is locally accessible in $x \in \mathbb{R}^{d}$ if for all $T>0$ the positive orbit up to time $T$

$$
\mathcal{O}_{\leq T}^{+}(x):=\{\varphi(t, x, u), 0<t \leq T \text { and } u \in \mathcal{U}\}
$$

and the negative orbit up to time $T$

$$
\mathcal{O}_{\leq T}^{-}(x):=\{\varphi(t, x, u),-T \leq t<0 \text { and } u \in \mathcal{U}\},
$$

have nonvoid interiors. It is called locally accessible in a subset $A \subset \mathbb{R}^{d}$ if it is locally accessible in every $x \in A$.

Local accessibility holds under a rank condition for the Lie algebra generated by the vector fields $f(\cdot, u), u \in U$; see, e.g., Jurdjevic [7]. We now define the main notion discussed in this paper; compare [4].

Definition 2.1 A subset $D$ of $\mathbb{R}^{d}$ with nonempty interior is a local control set if there exists a neighborhood $V$ of $\mathrm{cl} D$ such that for each $x, y \in D$ and every $\varepsilon>0$ there exist $T>0$ and $u \in \mathcal{U}$ such that

$$
\varphi(t, x, u) \in V \text { for all } t \in[0, T] \text { and } d(\varphi(T, x, u), y)<\varepsilon
$$

and for every $D^{\prime}$ with $D \subset D^{\prime} \subset V$ which satisfies this property, one has $D^{\prime}=D$.

The neighborhood $V$ in the definition above will also be called an isolating neighborhood of $D$. If the neighborhood $V$ can be chosen as $\mathbb{R}^{d}$, we obtain the usual notion of a control set with nonvoid interior as considered, e.g., in [2], Thus for local control sets the maximality property of control sets is replaced by a local maximality property, and we refer to the latter also as global control sets.

The following notation for sets $A, B \subset \mathbb{R}^{d}$ will be convenient.

$$
\mathcal{O}_{B}^{+}(A)=\left\{q \in \mathbb{R}^{d}, \quad \begin{array}{c}
\text { there are } T \geq 0, u \in \mathcal{U} \text { and } p \in A \text { with } \\
q=\varphi(T, p, u) \text { and } \varphi(t, p, u) \in B \text { for } 0 \leq t \leq T
\end{array}\right\}
$$

an analogous definition can be given for $\mathcal{O}_{B}^{-}(A)$. Thus a local control set with isolating neighborhood $N$ is a maximal subset $D$ of $N$ with nonvoid interior such that

$$
D \subset \operatorname{clO}_{N}^{+}(x) \text { for all } x \in D
$$


We state some facts on local control sets. First observe that there are at most countably many local control sets, since the topology has a countable base.

Proposition 2.2 Let $D$ be a local control set. Then

(i) $D$ is connected;

(ii) if local accessibility holds in a neighborhood of $\operatorname{cl} \operatorname{int} D$, then $\operatorname{cl} D=$ clint $D$.

If local accessibility holds on $\mathrm{cl} D$, then

(iii) int $D \subset \mathcal{O}^{+}(x)$ for all $x \in D$;

(iv) For an isolating neighborhood of $D$ and all $x \in \operatorname{int} D$ one has

$$
\operatorname{cl} \mathcal{O}_{N}^{+}(x) \cap \mathcal{O}_{N}^{-}(x)=D,
$$

(v) for every $x \in \operatorname{int} D$ there are $T>0$ and a T-periodic control function $u \in \mathcal{U}$ such that $\varphi(\cdot, x, u)$ is T-periodic and contained in $D$.

Proof. Assertions (i), (ii) and (v) are proven in [4]; assertions (iii) and (iv) follows as for control sets in Lemma 3.2.13 in [2].

We will need specific information on the boundary of local control sets.

Definition 2.3 For a local control set $D$ with isolating neighborhood $N$ define the following subsets of the boundary $\partial D$ :

$\partial^{e x} D=\left\{x \in \partial D\right.$, there is $y \in \operatorname{int} D$ with $\left.x \in \mathcal{O}_{N}^{+}(y)\right\}$,

$\partial^{e n} D=\left\{x \in \partial D\right.$, there is $y \in \operatorname{int} D$ with $\left.x \in \mathcal{O}_{N}^{-}(y)\right\}$,

$\partial^{t g} D=\left\{x \in \partial D, \mathcal{O}_{N}^{+}(x) \cap \operatorname{int} D=\emptyset\right.$ and $\left.\mathcal{O}_{N}^{-}(x) \cap \operatorname{int} D=\emptyset\right\}$.

These sets are called the exit, entrance, and tangential boundaries, respectively.

The following results are proven as [2, Lemma 3.2.22, Lemma 3.2.24 and Proposition 3.2.25], which are stated for global control sets only; however, the proofs are local and hence immediately apply to local control sets.

Proposition 2.4 Let $D$ be a local control set of system (1) such that local accessibility holds in int $D$. Then there exists a unique local control set $D^{*}$ of the time reversed system $\dot{x}(t)=-f(x(t), u(t)), u \in \mathcal{U}$, with $\operatorname{int} D=\operatorname{int} D^{*}$. 
Proposition 2.5 Let D be a local control set of system (1) such that local accessibility holds in $\mathrm{cl} D$.

(i) The three sets $\partial^{e n} D, \partial^{e x} D$, and $\partial^{t g} D$ form a decomposition of $\partial D$.

(ii) The sets $\partial^{e n} D$ and $\partial^{e x} D$ are open in $\partial D$ and $\partial^{t g} D$ is closed in $\partial D$.

(iii) The equality $\partial^{t g} D=\operatorname{cl} \partial^{e n} D \cap \operatorname{cl} \partial^{e x} D$ holds and $\operatorname{int}_{\partial D} \partial^{t g} D=\emptyset$.

For every point $x \in \partial D$ the following assertions hold:

(iv) $x \in \partial^{e n} D$ if and only if $x \in D$.

(v) $x \in \partial^{e x} D$ if and only if $x \in D^{*}$, where $D^{*}$ is the control set of the time reversed system from Lemma 2.4.

(vi) $x \in \partial^{t g} D$ if and only if $x \notin D \cup D^{*}$.

The following lemma shows that the closure of a local control set can be left and entered only finitely many times in finite time.

Lemma 2.6 Let $D$ be a local control set with $p_{0} \in \operatorname{int} D$ and consider a trajectory $x$ with $x(0)=x(T)=p_{0}$ for some $T>0$.

(i) There are only finitely many points $t_{i}^{+} \in[0, T]$ with $x\left(t_{i}^{+}\right) \in \partial^{e n} D$ and $x(t) \notin \mathrm{cl} D$ for all $t \in\left(t_{i}^{+}-\delta_{i}, t_{i}^{+}\right)$and some $\delta_{i}>0$.

(ii) There are only finitely many points $t_{i}^{-} \in[0, T]$ with $x\left(t_{i}^{-}\right) \in \partial^{e x} D$ and $x(t) \notin \mathrm{cl} D$ for all $t \in\left(t_{i}^{-}, t_{i}^{-}+\delta_{i}\right)$ and some $\delta_{i}>0$.

Proof. For assertion (i) consider $t_{i+1}^{+}>t_{i}^{+}$. Then $x\left(t_{i}^{+}\right), x\left(t_{i+1}^{+}\right) \in \partial^{e n} D \subset$ $D$. Since $x(t) \notin \mathrm{cl} D$ for all $t \in\left(t_{i+1}^{+}-\delta_{i+1}, t_{i+1}^{+}\right)$and some $\delta_{i+1}>0$, it follows that there is $s \in\left(t_{i}^{+}, t_{i+1}^{+}\right)$with $x(s) \notin \mathrm{cl} D$. By local maximality of $D$ we may assume that $x(s)$ is not in an isolating neighborhood of $\operatorname{cl} D$. By boundedness of the right hand side of (1), it follows that there can exist only finitely many points $t_{i}^{+}$. Assertion (ii) follows by time reversal.

The following lemma shows that the entrance boundary can only be left to the tangential boundary or the interior of the control set.

Lemma 2.7 Consider a local control set $D$.

(i) Let $x \in \partial^{\text {en }}(D)$ and suppose that for a control $u \in \mathcal{U}$ one has $\varphi(T, x, u) \notin$ $\partial^{\text {en }}(D)$ for some $T>0$. Then there is $\tau \geq 0$ such that either $\varphi(t, x, u) \in$ $\partial^{e n} D$ for all $t \in[0, \tau)$ and $\varphi(\tau, x, u) \in \partial^{t g} D$ or $\varphi(t, x, u) \in \partial^{e n} D$ for all $t \in[0, \tau]$ and $\varphi(t, x, u) \in \operatorname{int} D$ for all $t-\tau>0$, small.

(ii) Let $x \in \partial^{e x}(D)$ and suppose that for a control $u \in \mathcal{U}$ one has $\varphi(T, x, u) \notin$ $\partial^{e x}(D)$ for some $T>0$. Then there is $\tau \geq 0$ such that either $\varphi(t, x, u) \in \partial^{e x} D$ for all $t \in[0, \tau)$ and $\varphi(\tau, x, u) \in \partial^{t g} D$ or $\varphi(t, x, u) \in \partial^{\text {ex } D}$ for all $t \in[0, \tau]$ and $\varphi(t, x, u) \notin \operatorname{cl} D$ for all $t-\tau>0$, small. 
Proof. (i) Let $\tau=\sup \left\{t \geq 0, \varphi(s, x, u) \in \partial^{e n} D\right.$ for all $\left.s \in[0, t)\right\}$. Since $\partial D$ is closed it follows that $\varphi(\tau, x, u) \in \partial D$. Since $\partial^{e n} D$ and $\partial^{e x} D$ are open in $\partial D$ and disjoint, it follows that either $\varphi(\tau, x, u) \in \partial^{t g} D$ or $\varphi(\tau, x, u) \in \partial^{e n} D$. It remains to discuss the second case. For arbitrarily small $t-\tau>0$ one has $\varphi(t, x, u) \notin \partial^{e n} D$. Since $\varphi(\tau, x, u) \in \partial^{e n} D$, this implies for these $t$ that $\varphi(t, x, u) \in \operatorname{int} D$. In fact, otherwise it follows that $\varphi(t, x, u) \notin \operatorname{cl} D$ and, by continuous dependence on the initial value, a neighborhood of $\varphi(\tau, x, u)$ is mapped into the complement of $\operatorname{cl} D$. Hence arbitrarily close to $\varphi(\tau, x, u)$ there are points in $\partial^{e x} D$, which is impossible, since $\varphi(\tau, x, u)$ has a positive distance to $\partial^{e x} D$. Now consider $t_{i} \downarrow \tau$ with $\varphi\left(t_{i}, x, u\right) \in \operatorname{int} D$. If there are $s_{i} \downarrow \tau$ with $\varphi\left(s_{i}, x, u\right) \notin \operatorname{int} D$, then the trajectory leaves $\operatorname{int} D$ between $s_{i+1}$ and $s_{i}$. This is only possible through $\partial^{e x} D$. By Lemma 2.6 this is only possible finitely often. Hence $\varphi(t, x, u) \in \operatorname{int} D$ for all $t-\tau>0$, small.

(ii) follows analogously.

\section{Operations on local control sets}

It is clear that, in general, neither the union nor the intersection of local control sets is a local control set. We will now discuss two constructions, similar to the union and the intersection, that when applied to local control sets yield an element of the same class.

Concerning the union of local control sets, it may happen that $D \cup D^{\prime}$ is not a local control set because it is not maximal. Hence we have to enlarge the union appropriately to get a local control set. We impose a "regularity" condition requiring (locally) that $\mathcal{O}^{+}(\mathrm{cl} D) \cap \mathcal{O}^{-}\left(\mathrm{cl} D^{\prime}\right)$ is closed. This, in the case of control affine systems with compact control range, means that there are no points which can only be reached in infinite times forward from $\mathrm{cl} D$ and backward from $\mathrm{cl} D^{\prime}$.

Proposition 3.1 Let $D$ and $D^{\prime}$ be local control sets with with isolating neighborhoods $N$ and $N^{\prime}$, respectively. Consider the set

$$
W=N \cup N^{\prime}
$$

and suppose that int $D \cap \operatorname{int} D^{\prime} \neq \varnothing$. Assume that the sets

$$
\mathcal{O}_{W}^{+}(\operatorname{cl} D) \cap \mathcal{O}_{W}^{-}\left(\operatorname{cl} D^{\prime}\right) \text { and } \mathcal{O}_{W}^{+}\left(\operatorname{cl} D^{\prime}\right) \cap \mathcal{O}_{W}^{-}(\operatorname{cl} D)
$$


are closed. Then the set

$$
E=\left[\mathcal{O}_{W}^{+}(D) \cap \mathcal{O}_{W}^{-}\left(D^{\prime}\right)\right] \cup\left[\mathcal{O}_{W}^{+}\left(D^{\prime}\right) \cap \mathcal{O}_{W}^{-}(D)\right]
$$

is a local control set with isolating neighborhood $W$.

Proof. Note that $D \cup D^{\prime} \subset E$, since $\operatorname{int} D \cap \operatorname{int} D^{\prime} \neq \varnothing$ implies that

$$
D \subset \mathcal{O}_{W}^{+}(D) \cap \mathcal{O}_{W}^{-}\left(D^{\prime}\right) \text { and } D^{\prime} \subset \mathcal{O}_{W}^{+}\left(D^{\prime}\right) \cap \mathcal{O}_{W}^{-}(D) \text {. }
$$

Next observe that

$$
\begin{aligned}
\operatorname{cl} E & =\operatorname{cl}\left(\left[\mathcal{O}_{W}^{+}(D) \cap \mathcal{O}_{W}^{-}\left(D^{\prime}\right)\right] \cup\left[\mathcal{O}_{W}^{+}\left(D^{\prime}\right) \cap \mathcal{O}_{W}^{-}(D)\right]\right) \\
& =\operatorname{cl}\left[\mathcal{O}_{W}^{+}(D) \cap \mathcal{O}_{W}^{-}\left(D^{\prime}\right)\right] \cup \operatorname{cl}\left[\mathcal{O}_{W}^{+}\left(D^{\prime}\right) \cap \mathcal{O}_{W}^{-}(D)\right] \\
& \subset \operatorname{cl}\left[\mathcal{O}_{W}^{+}(\operatorname{cl} D) \cap \mathcal{O}_{W}^{-}\left(\operatorname{cl} D^{\prime}\right)\right] \cup \operatorname{cl}\left[\mathcal{O}_{W}^{+}\left(\operatorname{cl} D^{\prime}\right) \cap \mathcal{O}_{W}^{-}(\operatorname{cl} D)\right] \\
& =\left[\mathcal{O}_{W}^{+}(\operatorname{cl} D) \cap \mathcal{O}_{W}^{-}\left(\operatorname{cl} D^{\prime}\right)\right] \cup\left[\mathcal{O}_{W}^{+}\left(\operatorname{cl} D^{\prime}\right) \cap \mathcal{O}_{W}^{-}(\operatorname{cl} D)\right]
\end{aligned}
$$

the last equality follows from our assumption. In order to show that $W$ is a neighborhood of $\operatorname{cl} E$ take $x \in \mathcal{O}_{W}^{+}(\operatorname{cl} D) \cap \mathcal{O}_{W}^{-}\left(\operatorname{cl} D^{\prime}\right)$. Thus there are $T>0$, $u \in \mathcal{U}$ and $y \in \operatorname{cl} D$ with

$$
x=\varphi(T, y, u) \text { and } \varphi(t, x, u) \in W \text { for } 0 \leq t \leq T .
$$

By continuous dependence on final values and $y \in \operatorname{int} N$ it follows that

$$
x \in \operatorname{int} \varphi(T, N, u) \subset W .
$$

Analogously one argues for $x \in \mathcal{O}_{W}^{+}\left(\operatorname{cl} D^{\prime}\right) \cap \mathcal{O}_{W}^{-}(\operatorname{cl} D)$. Next for approximate controllability consider $x, y \in E$. Consider first

$$
x \in \mathcal{O}_{W}^{+}(D) \cap \mathcal{O}_{W}^{-}\left(D^{\prime}\right) \text { and } y \in \mathcal{O}_{W}^{+}\left(D^{\prime}\right) \cap \mathcal{O}_{W}^{-}(D) .
$$

First steer $x$ to a point $z_{1}$ in $D^{\prime}$ and a point $z_{2}$ in $D^{\prime}$ to $y$. One can also steer $z_{1}$ to $z_{2}$. All three trajectories and hence their concatenation steering the system from $x$ to $y$ may be chosen in $W$. Next consider

$$
x, y \in \mathcal{O}_{W}^{+}(D) \cap \mathcal{O}_{W}^{-}\left(D^{\prime}\right) .
$$

First steer $x$ to a point $z_{1}$ in $D^{\prime}$, and a point $z_{2}$ in $D$ to $y$. One can also approximately steer $z_{1}$ to $z_{2}$, using $\mathcal{O}_{W}^{+}\left(D^{\prime}\right) \cap \mathcal{O}_{W}^{-}(D) \neq \varnothing$ and approximate controllability in $D$ and $D^{\prime}$. Using continuous dependence on initial 
values one can steer $x$ approximately to $y$. All trajectories and hence their concatenation may be chosen in $W$. Similarly one can treat the other cases

$$
x \in \mathcal{O}_{W}^{+}\left(D^{\prime}\right) \cap \mathcal{O}_{W}^{-}(D) \text { and } y \in \mathcal{O}_{W}^{+}(D) \cap \mathcal{O}_{W}^{-}\left(D^{\prime}\right) .
$$

and

$$
x, y \in \mathcal{O}_{W}^{+}\left(D^{\prime}\right) \cap \mathcal{O}_{W}^{-}(D) .
$$

It remains to show that $E$ is maximal in $N \cup N^{\prime}$. So let $E^{\prime}$ be a set containing $E$ such that every $x$ can approximately be controlled to any other $y \in E^{\prime}$ within $W$. Choosing $y \in \operatorname{int} D^{\prime}$ one finds that $x \in \mathcal{O}_{W}^{-}\left(D^{\prime}\right)$. Similarly, one sees that a point $z \in D$ can be steered to $x$, and hence $x \in \mathcal{O}_{W}^{+}(D) \cap \mathcal{O}_{W}^{-}\left(D^{\prime}\right) \subset E$. (The same argument also shows that $x \in \mathcal{O}_{W}^{+}\left(D^{\prime}\right) \cap \mathcal{O}_{W}^{-}(D)$ ). We conclude that $E=E^{\prime}$.

Proposition 3.2 Assume that the number of local control sets is finite and that, for $n \in \mathbb{N}$, there are neighborhoods $N_{n}$ and $N_{n}^{\prime}$ of $D$ and $D^{\prime}$, respectively, with

$$
\begin{aligned}
& N_{n} \supset N_{n+1} \quad \text { and } \quad N_{n}^{\prime} \supset N_{n+1}^{\prime} \quad \text { for } n \in \mathbb{N} \text {, } \\
& \bigcap_{n} N_{n}=\operatorname{cl} D \quad \text { and } \bigcap_{n} N_{n}^{\prime}=\operatorname{cl} D^{\prime}
\end{aligned}
$$

such that, for $W_{n}=N_{n} \cup N_{n}^{\prime}$, the sets

$$
\mathcal{O}_{W_{n}}^{+}(\operatorname{cl} D) \cap \mathcal{O}_{W_{n}}^{-}\left(\operatorname{cl} D^{\prime}\right) \quad \text { and } \quad \mathcal{O}_{W_{n}}^{+}\left(\operatorname{cl} D^{\prime}\right) \cap \mathcal{O}_{W_{n}}^{-}(\operatorname{cl} D)
$$

are closed. Then there exists a unique local control set which we denote by $D \sqcup D^{\prime}$ with

$$
D \cup D^{\prime} \subset D \sqcup D^{\prime} \subset \operatorname{cl}\left(D \cup D^{\prime}\right)
$$

Proof. For the isolating neighborhoods $N_{n}$ and $N_{n}^{\prime}$ we can construct a local control set $E_{n}$ with isolating neighborhood $N_{n} \cup N_{n}^{\prime}$ as in the previous proposition. Then, clearly, for $N_{n} \subset N_{m}$ and $N_{n}^{\prime} \subset N_{m}^{\prime}$ one has $D \cup D^{\prime} \subset$ $E_{n} \subset E_{m} \subset N_{m} \cup N_{m}$. Since, by assumption, the number of local control sets is finite, we conclude that for some $N \in \mathbb{N}$ all $E_{n}, n>N$, coincide and we denote this local control set by $D \sqcup D^{\prime}$. Clearly, it satisfies (5).

We now study the intersection of local control sets $D$ and $D^{\prime}$. One easily observes that $D \cap D^{\prime}$ need not be a local control set, since the trajectories 
between points $p$ and $q$ in $D \cap D^{\prime}$ need not be contained in this set (they do exist in $D$ and in $D^{\prime}$ ).

Let $N$ and $N^{\prime}$ be isolating neighborhoods of $D$ and $D^{\prime}$. A natural candidate for a notion of 'intersection' of $D$ and $D^{\prime}$ is a maximal local control set in $N \cap N^{\prime}$. Unfortunately, it may happen that $N \cap N^{\prime}$ may be empty or contain more than one local control set.

Proposition 3.3 Let $D$ and $D^{\prime}$ be local control sets with with isolating neighborhoods $N$ and $N^{\prime}$, respectively, and let $W=N \cap N^{\prime}$. Then

$$
D \wedge D^{\prime}:=\bigcup_{p \in D \cap D^{\prime}}\left[\operatorname{cl} \mathcal{O}_{W}^{+}(p) \cap \mathcal{O}_{W}^{-}(p)\right]
$$

is the union of local control sets with isolating neighborhood $W$ (here also the empty set is allowed).

Proof. Note that $D \wedge D^{\prime} \subset D \cap D^{\prime}$, since all points in $\left[D, D^{\prime}\right]_{W}$ are in $D$ :

$$
\operatorname{cl}_{W}^{+}(p) \cap \mathcal{O}_{W}^{-}(p) \subset \operatorname{cl} \mathcal{O}_{N}^{+}(p) \cap \mathcal{O}_{N}^{-}(p)=D .
$$

They are also in $D^{\prime}$ and hence in the intersection. The set $W$ is a neighborhood of $\mathrm{cl}\left(D \wedge D^{\prime}\right)$, since

$$
\operatorname{cl}\left(D \wedge D^{\prime}\right) \subset \operatorname{cl}\left(D \cap D^{\prime}\right) \subset \operatorname{cl} D \cap \operatorname{cl} D^{\prime} \subset N \cap N^{\prime}=W .
$$

If, for some $p \in D \cap D^{\prime}$ the intersection $\operatorname{cl}^{+} \mathcal{O}_{W}^{+}(p) \cap \mathcal{O}_{W}^{-}(p)$ is nonvoid, then it is a maximal set such that complete approximate controllability within $W$ holds. Using continuous dependence on initial conditions one sees that it is in fact a maximal set with this property.

When $D \wedge D^{\prime}$ contains only one local control set, it coincides with it. We will study the case where this local control set coincides up to closure with the intersection of $D$ and $D^{\prime}$ and give the following formal definition for the intersection and the union of local control sets.

Definition 3.4 Let $D$ and $D^{\prime}$ be local control sets. If there exists a local control set, denoted by $D \sqcup D^{\prime}$, with

$$
D \cup D^{\prime} \subset D \sqcup D^{\prime} \subset \operatorname{cl}\left(D \cup D^{\prime}\right),
$$

it is called the union of $D$ and $D^{\prime}$. If there exists a local control set, denoted by $D \sqcap D^{\prime}$, with

$$
D \sqcap D^{\prime} \subset D \cap D^{\prime} \text { and } \operatorname{cl}\left(D \sqcap D^{\prime}\right)=\operatorname{cl}\left(D \cap D^{\prime}\right)=\operatorname{cl} D \cap \operatorname{cl} D^{\prime},
$$

it is called the intersection of $D$ and $D^{\prime}$. 
Note that Proposition 3.2 gives a sufficient condition for the existence of $D \sqcup D^{\prime}$. The local control set $D \sqcap D^{\prime}$ has the isolating neighborhood $N \cap N^{\prime}$, where $N$ and $N^{\prime}$ are isolating neighborhoods of $D$ and $D^{\prime}$, respectively.

\section{The fundamental semigroup}

Consider a local control set $D \subset \mathbb{R}^{d}$ of system (1) and fix $p_{0} \in \operatorname{int} D$. Define

$$
P\left(D, p_{0}\right)=\left\{\begin{array}{cc} 
& x(0)=x(1)=p_{0}, x(t) \in \operatorname{int} D \text { for } \\
x \in W^{1, \infty}\left([0,1], \mathbb{R}^{d}\right), & t \in[0,1] \text { and } \exists \gamma_{x}^{-}, \gamma_{x}^{+}>0 \text { s.t. } \\
\dot{x}(t) \in\left[\gamma_{x}^{-}, \gamma_{x}^{+}\right] f(x(t), U), t \in[0,1]
\end{array}\right\}
$$

We endow $P\left(D, p_{0}\right)$ with the metric structure given by $d\left(x_{1}, x_{2}\right)=\left\|x_{1}-x_{2}\right\|_{\infty}$ for $x_{1}, x_{2} \in P\left(D, p_{0}\right)$.

The following result characterizes the elements of $P\left(D, p_{0}\right)$ as reparametrized trajectories of the control system. Note the role of the lower bound $\gamma_{x}^{-}>0$ which appears in the definition of $P\left(D, p_{0}\right)$; if it is absent, every point, in particular, the point $p_{0}$, is an equilibrium for the system in (8). The theorem shows that $P\left(D, p_{0}\right)$ consists of the periodic trajectories in $\operatorname{int} D$ of (1) through $p_{0}$, reparametrized to $[0,1]$.

Theorem 4.1 Let $x \in W^{1, \infty}\left([0,1], \mathbb{R}^{d}\right)$ be such that $x(0)=x(1)=p_{0}$ and $x(t) \in \operatorname{int} D$ for $t \in[0,1]$. Then $x \in P\left(D, p_{0}\right)$ if and only if there are measurable functions $\gamma:[0,1] \rightarrow\left[\gamma_{x}^{-}, \gamma_{x}^{+}\right]$and $u:[0,1] \rightarrow U$ such that

$$
\dot{x}(t)=\gamma(t) f(x(t), u(t)), \quad t \in[0,1] .
$$

Equivalently, for $\alpha(s)=\int_{0}^{s} \gamma(t) d t, s \in[0,1]$ and $T=\int_{0}^{1} \gamma(t) d t$, the function $y(t):=x(\alpha(t))$ is a T-periodic solution in $\operatorname{int} D$ of (1) with $y(0)=p_{0}$.

Proof. The latter equivalence is clear since it based on a time reparametrization. Observe that

$$
\frac{d}{d s} \alpha(s)=\gamma(s), \quad s \in[0,1],
$$

and hence

$$
\frac{d}{d t} \alpha^{-1}(t)=\frac{1}{\gamma(t)}, \quad t \in[0, T]
$$


It is also clear that a solution of (8) is an element of $P\left(D, p_{0}\right)$. Consider $x \in W^{1, \infty}\left([0,1], \mathbb{R}^{d}\right)$ with $x(0)=x(1)=p_{0}$ and $x(t) \in \operatorname{int} D$ for $t \in[0,1]$ and

$$
\dot{x}(t) \in\left[\gamma_{x}^{-}, \gamma_{x}^{+}\right] f(x(t), U) \text { for a.a. } t \in[0,1] \text { and } \gamma_{x}^{-}, \gamma_{x}^{+}>0 .
$$

Since $f$ is $C^{1}$, it is a Carathéodory map. Thus by Filippov's Theorem, see e.g. Aubin/Frankowska [1], Theorem 8.2.10, there exists a measurable function $[0,1] \rightarrow\left[\gamma_{x}^{-}, \gamma_{x}^{+}\right] \times U$ given by $t \mapsto(\gamma(t), u(t))$ such that

$$
\dot{x}(t)=\gamma(t) f(x(t), u(t)) \quad \text { for a.a. } t \in[0,1] .
$$

This concludes the proof.

As a consequence, we find that for a local control set $D$ with local accessibility in $D$, one has $P\left(D, p_{0}\right) \neq \emptyset$ for all $p_{0} \in \operatorname{int} D$.

Given $x, y \in P\left(D, p_{0}\right)$ we say that they are homotopic, written $x \simeq y$, if there exists a continuous map (a homotopy) $H:[0,1] \rightarrow P\left(D, p_{0}\right)$ such that $H(0)=x$ and $H(1)=y$. One can check that this is an equivalence relation. We will denote by $\Lambda\left(D, p_{0}\right)$ the quotient $P\left(D, p_{0}\right) / \simeq$.

Define a binary operation on $P\left(D, p_{0}\right)$ by setting for $x, y \in P\left(D, p_{0}\right)$

$$
(x * y)(t)= \begin{cases}x(2 t) & t \in[0,1 / 2] \\ y(2 t-1) & t \in[1 / 2,1]\end{cases}
$$

Clearly, the map $P\left(D, p_{0}\right) \times P\left(D, p_{0}\right) \rightarrow P\left(D, p_{0}\right)$ given by $(x, y) \mapsto x * y$ is continuous.

We extend this operation to $\Lambda\left(D, p_{0}\right)$ by passing to the quotient, i.e., we set $[x] *[y]:=[x * y]$ where $x, y \in P\left(D, p_{0}\right)$ and the square brackets denote equivalence classes. We now show that the operation is well defined in $\Lambda\left(D, p_{0}\right)$, that is, it does not depend on the chosen representative for the equivalence class. Assume $x \simeq x^{\prime}$ and $y \simeq y^{\prime}$ with homotopies $h_{1}$ and $h_{2}$ respectively. Then $h_{1} * h_{2}$ yields a homotopy between $x * y$ and $x^{\prime} * y^{\prime}$; in other words $[x * y]=\left[x^{\prime} * y^{\prime}\right]$.

Notice that ' $*$ ' is not associative on $P\left(D, p_{0}\right)$, however, it turns out to be associative on $\Lambda\left(D, p_{0}\right)$. To see that, let $x, y, z \in P\left(D, p_{0}\right)$ and notice that $\lambda \mapsto h_{\lambda}(\cdot)$, with

$$
h_{\lambda}(t)= \begin{cases}x(2 t+2 \lambda t) & t \in\left[0, \frac{1}{2}-\frac{\lambda}{4}\right] \\ y(4 t-2-\lambda) & t \in\left[\frac{1}{2}-\frac{\lambda}{4}, \frac{3}{4}-\frac{\lambda}{4}\right] \\ z(4 t-2 \lambda t+2 \lambda-3) & t \in\left[\frac{3}{4}-\frac{\lambda}{4}, 1\right]\end{cases}
$$

yields a homotopy between $x *(y * z)$ and $(x * y) * z$. 
Remark 4.2 Observe that this map is not continuous with respect to the $W^{1, \infty}$-topology which at first glance might appear more natural for $P\left(D, p_{0}\right)$.

Definition 4.3 For a local control set $D$ and $p_{0} \in \operatorname{int} D$ the semigroup $\Lambda\left(D, p_{0}\right)$ with the operation ' $*$ ' is called the fundamental semigroup of the pointed local control set $\left(D, p_{0}\right)$.

Remark 4.4 As already noticed the operation ' $*$ ' is continuous in $P\left(D, p_{0}\right)$. Thus the semigroup $\Lambda\left(D, p_{0}\right)$ is, in fact, a topological semigroup (with the quotient topology). This topology, however, does not seem to carry interesting information.

In general, the fundamental semigroup does not admit a unity, however a unity exists when $p_{0}$ is an equilibrium. Note that the unity in a semigroup is unique (we refer to Howie [6] for the general theory of algebraic semigroups).

Proposition 4.5 If $p_{0} \in \operatorname{int} D$ is an equilibrium for the control system, then the function $x_{0}(t) \equiv p_{0}$ represents the unity of $\Lambda\left(D, p_{0}\right)$.

Proof. We need to prove that $x_{0} * x \simeq x * x_{0} \simeq x$ for any $x \in P\left(D, p_{0}\right)$. Consider the map $H:[0,1] \rightarrow P\left(D, p_{0}\right)$ given by $\lambda \mapsto h_{\lambda}(\cdot)$ with

$$
h_{\lambda}(t)= \begin{cases}p_{0} & t \in\left[0, \frac{1-\lambda}{2}\right] \\ x(2 t-t \lambda+\lambda-1) & t \in\left[\frac{1-\lambda}{2}, 1\right]\end{cases}
$$

Clearly $H$ is a homotopy between $x_{0} * x$ and $x$. An analogous homotopy can be constructed between $x * x_{0}$ and $x$.

Remark 4.6 Let $x$ and $y$ be elements of $P\left(D, p_{0}\right)$ that differ only by a reparametrization, i.e., there exists a continuous bijective map $\tau:[0,1] \rightarrow$ $[0,1]$, with $\tau(0)=0$ and $\tau(1)=1$, such that $x(\tau(t))=y(t)$ for $t \in[0,1]$. Then $x \simeq y$ since

$$
h(\lambda, t)=x(\lambda \tau(t)+(1-\lambda) t)
$$

defines a homotopy, since $h(\lambda, \cdot)$ belongs to $P\left(D, p_{0}\right)$ and $h$ is continuous.

For linear control systems, the fundamental semigroup is trivial as shown by the following proposition. 
Proposition 4.7 Consider the linear control system

$$
\dot{x}=A x+B u, \quad u \in \mathcal{U},
$$

with $A \in \mathbb{R}^{d \times d}$ and $B \in \mathbb{R}^{d \times m}$. Assume that $(A, B)$ is controllable and $U$ is compact, convex with $0 \in \operatorname{int} U$. Then there exists a unique local control set $D$, and the semigroup $\Lambda(D, 0)$ consists of just its unity.

Proof. By Example 3.2.16 in [2] there exists a unique control set $D$ and it contains the origin in its interior. By linearity the set $P(D, 0)$ is convex and this yields the desired homotopy. By Theorem 4.1 this is also a homotopy of periodic trajectories through $p_{0}=0$ implying that the control set $D$ is also the unique local control set.

The following simple example shows that the fundamental semigroup allows us to distinguish control sets via the dynamic behavior of the system.

Example 4.8 Let $U \subset \mathbb{R}^{m}$ be a compact and convex set containing 0 in its interior. Consider control-affine systems of the form

$$
\dot{x}=f_{0}(x)+\sum_{i=1}^{m} u_{i}(t) f_{i}(x), u \in \mathcal{U}^{\rho},
$$

where $\mathcal{U}^{\rho}$ denotes the set of measurable functions on $\mathbb{R}$ with values in $\rho U$. Suppose that the uncontrolled system (with $u \equiv 0$ ) has a homoclinic orbit given by

$$
\varphi\left(t, p_{1}, 0\right), t \in \mathbb{R}, \text { with } \lim _{t \rightarrow \pm \infty} \varphi\left(t, p_{1}, 0\right)=p_{0},
$$

where $p_{0} \neq p_{1}$ is an equilibrium. Suppose that $H:=\left\{p_{0}\right\} \cup\left\{\varphi\left(t, p_{1}, 0\right), t \in\right.$ $\mathbb{R}\}$ is a chain recurrent component of the uncontrolled system and that the controllability condition

$$
\operatorname{span}\left\{\operatorname{ad}_{f_{0}}^{k} f_{i}(x), i=1, \ldots, m, k=0,1, \ldots\right\}=\mathbb{R}^{d}
$$

holds for all points $x \in H$. Then for every $\rho>0$ there is a control set $D^{\rho}$ containing $H$ in its interior and

$$
\bigcap_{\rho>0} D^{\rho}=H
$$

see Corollary 4.7.6 in [2] (the controlled Takens-Bogdanov oscillator is a system where these conditions can be verified; cp. Häckl/Schneider [5] or Section 9.4 in [2]). By Proposition 4.5 the fundamental semigroup $\Lambda^{\rho}\left(D^{\rho}, p_{0}\right)$ 
contains a unity. On the other hand, consider a system (10) where the uncontrolled system has a periodic trajectory $\hat{H}=\left\{\varphi\left(t, p_{0}, 0\right), t \in[0, T]\right\}$. If $\hat{H}$ is a chain recurrent component of the uncontrolled system and (11) holds on $\hat{H}$, then again Corollary 4.7.6 in [2] implies the existence of control sets $\hat{D}^{\rho}$ containing $\hat{H}$ in the interiors with $\bigcap_{\rho>0} \hat{D}^{\rho}=\hat{H}$. We will show that for $\rho>0$, small enough, the fundamental semigroups $\Lambda^{\rho}\left(\hat{D}^{\rho}, p_{0}\right)$ do not contain a unity. In fact, let $\rho>0$ be small enough, such that there is no periodic trajectory through $p_{0}$ which is contractible in int $\hat{D}^{\rho}$. Concatenation of the periodic trajectory with itself yields existence of elements $a \in \Lambda^{\rho}\left(\hat{D}^{\rho}, p_{0}\right)$ with $a^{n} \neq a^{m}$ for $m, n \in \mathbb{N}$ with $m \neq n$. Suppose that there exists an element $e \in \Lambda^{\rho}\left(\hat{D}^{\rho}, p_{0}\right)$ with $e \circ a=a$. Then periodic trajectories representing a and $b=e \circ a$ yield elements $a^{\prime}$ and $b^{\prime}$ in the fundamental group of int $\hat{D}^{\rho}$. Since $e \circ a$ is a concatenation, we get a decomposition $b^{\prime}=e^{\prime} \circ a^{\prime}$ in the fundamental group with $e^{\prime}$ representing $e$. Since e o $a=a$ in $\Lambda^{\rho}\left(\hat{D}^{\rho}, p_{0}\right)$, one has

$$
e^{\prime} \circ a^{\prime}=b^{\prime}=a^{\prime}
$$

in the fundamental group. This implies that $e^{\prime}$ is the unity in the fundamental group. Since there is no periodic trajectory which is contractible in $\operatorname{int} \hat{D}^{\rho}$, this is a contradiction.

Now we analyze the dependence of the constructed semigroups on the underlying local control sets. Let $D \subset D^{\prime}$ be local control sets for (1). If $p_{0} \in$ int $D$, the inclusion $i: D \hookrightarrow D^{\prime}$ induce a homomorphism $i_{*}: \Lambda\left(D, p_{0}\right) \rightarrow$ $\Lambda\left(D^{\prime}, p_{0}\right)$ which maps $[x] \in \Lambda\left(D, p_{0}\right)$ to the element $[x]$ of $\Lambda\left(D^{\prime}, p_{0}\right)$.

Lemma 4.9 Take $x \in P\left(D^{\prime}, p_{0}\right) \backslash P\left(D, p_{0}\right)$. Then $y \simeq x$ implies $y \in$ $P\left(D^{\prime}, p_{0}\right) \backslash P\left(D, p_{0}\right)$.

Proof. By the definition of local control sets, there exists an open neighborhood $V$ of $p_{0}$ containing cl $D$ such that $D$ is the maximal subset of complete controllability of $V$ (and, clearly, we may assume that $D^{\prime} \not \subset V$ ). Assume by contradiction that $x \in P\left(D, p_{0}\right)$. Since $y \simeq x$, there exists a continuous map $H:[0,1] \rightarrow P\left(D^{\prime}, p_{0}\right), \lambda \mapsto x_{\lambda}$, such that $H(0)=x$ and $H(1)=y$. Therefore, by continuity, there exists some $\lambda_{0} \in[0,1]$ such that $x_{\lambda_{0}}([0,1]) \subset V$ and $x_{\lambda_{0}}([0,1]) \not \subset D$. By Theorem 4.1 the set

$$
x_{\lambda_{0}}([0,1]) \cup D \subset V
$$

is completely controllable contradicting the choice of $V$. 
Proposition 4.10 Let $D$ and $D^{\prime}$ be local control sets for (1) such that $p_{0} \in$ $\operatorname{int} D$ and $D \subset D^{\prime}$. Then $i_{*}$ is injective and, if $D \neq D^{\prime}$, then $i_{*}$ is not surjective.

Proof. We may assume that $D \neq D^{\prime}$. To prove that $i_{*}$ is injective we have to show is that given any $x$ and $y$ in $P\left(D, p_{0}\right)$, with $[x] \neq[y]$ in $\Lambda\left(D, p_{0}\right)$, they cannot be joined by a continuous curve in $P\left(D^{\prime}, p_{0}\right)$. In fact, if they are connected by some $H:[0,1] \rightarrow P\left(D^{\prime}, p_{0}\right), \lambda \mapsto x_{\lambda}$, there exists $\lambda_{0} \in[0,1]$ such that $x_{\lambda_{0}} \in P\left(D^{\prime}, p_{0}\right) \backslash P\left(D, p_{0}\right)$, but this is impossible by Lemma 4.9. By Lemma 4.9 , no element of $P\left(D^{\prime}, p_{0}\right) \backslash P\left(D, p_{0}\right)$ can be joined to any one of $P\left(D, p_{0}\right)$ by a continuous curve in $P\left(D^{\prime}, p_{0}\right)$. This means that, given any $x \in P\left(D^{\prime}, p_{0}\right) \backslash P\left(D, p_{0}\right)$ one has $[x] \notin i_{*}\left(\Lambda\left(D, p_{0}\right)\right)$. Hence $i_{*}$ is not surjective.

Theorem 4.10 allows us to drop the $i_{*}$ and consider $\Lambda\left(D, p_{0}\right)$ as a subsemigroup of $\Lambda\left(D^{\prime}, p_{0}\right)$.

When $D \varsubsetneqq D^{\prime}$, Theorem 4.10 means that $\Lambda\left(D, p_{0}\right)$ is a proper subsemigroup of $\Lambda\left(D, p_{0}\right)$. We note the following immediate consequence of Theorem 4.10 .

Corollary 4.11 Let $D^{l o c}$ be a local control set for (1) and let $D$ be a (global) control set containing $p_{0}$ in its interior. If $D^{l o c} \neq D$, then $\Lambda\left(D^{l o c}, p_{0}\right)$ is a proper subsemigroup of $\Lambda\left(D, p_{0}\right)$.

Theorem 4.10 says that one cannot pass from a local control set to a larger one containing it without a 'jump' in the complexity of the associated semigroup. In particular, one cannot have nested local control sets with the same associated semigroup.

A similar argument shows that one can identify the 'minimal' local control set containing a given equilibrium.

Theorem 4.12 Let $D$ be a local control set for (1) containing the equilibrium $p_{0}$. If $\Lambda\left(D, p_{0}\right)$ consists only of its unity, then every local control set containing $p_{0}$ must contain D.

Proof. Assume by contradiction that there exists a local control set $D^{\prime}$ containing $p_{0}$ and such that $D \cap D^{\prime} \varsubsetneqq D$. Let $V^{\prime}$ be an isolating neighborhood of $D^{\prime}$. One can find an element $x \in P\left(D, p_{0}\right) \backslash P\left(D^{\prime}, p_{0}\right)$. By assumption, $\Lambda\left(D, p_{0}\right)$ consists only of its unity. Therefore $\Lambda\left(D, p_{0}\right)=\left\{\left[x_{0}\right]\right\}$, with $x_{0}(t) \equiv$ $p_{0}$. Consequently there exists a continuous $H:[0,1] \mapsto P\left(D, p_{0}\right), H(\lambda)=x_{\lambda}$, 
with $H(0)=x$ and $H(1)=x_{0}$. Then there exists some $\bar{\lambda} \in[0,1]$ such that $x_{\bar{\lambda}}([0,1]) \subset V^{\prime}$ and $x_{\bar{\lambda}}([0,1]) \not \subset D^{\prime}$. This, as in the proof of Lemma 4.9, yields a contradiction.

Proposition 4.13 Let $D \subset D^{\prime}$ be local control sets. If for some $p_{0} \in \operatorname{int} D$ the fundamental semigroup $\Lambda\left(D^{\prime}, p_{0}\right)$ has a unity, then also $\Lambda\left(D, p_{0}\right)$ has a unity.

Proof. Let $x_{0} \in P\left(D^{\prime}, p_{0}\right)$ be such that $\left[x_{0}\right]$ is the unity in $\Lambda\left(D^{\prime}, p_{0}\right)$. Then for every $[y] \in \Lambda\left(D, p_{0}\right) \subset \Lambda\left(D^{\prime}, p_{0}\right)$ one has $y * x_{0} \simeq y$ in $P\left(D^{\prime}, p_{0}\right)$..If $x_{0} \notin P\left(D, p_{0}\right)$, then $y * x_{0} \in P\left(D^{\prime}, p_{0}\right) \backslash P\left(D, p_{0}\right)$. Now Lemma 4.9 yields a contradiction. Hence $x_{0} \in P\left(D, p_{0}\right)$ and obviously, it represents the unity in $\Lambda\left(D, p_{0}\right)$.

In the next section we will study semigroups for the intersection and union of local control sets $D$ and $D^{\prime}$ satisfying (6) and (7), respectively, as specified in Definition 3.4. Then consider the inclusion maps

$$
\begin{array}{ll}
i: D \sqcap D^{\prime} \rightarrow D, & i^{\prime}: D \sqcap D^{\prime} \rightarrow D^{\prime}, \\
j: D \rightarrow D \sqcup D^{\prime}, & j^{\prime}: D^{\prime} \rightarrow D \sqcup D^{\prime} .
\end{array}
$$

and, for $p_{0} \in \operatorname{int}\left(D \sqcap D^{\prime}\right)$, the corresponding semigroups

$$
\Lambda\left(D \sqcap D^{\prime}, p_{0}\right), \Lambda\left(D, p_{0}\right), \Lambda\left(D^{\prime}, p_{0}\right), \Lambda\left(D \sqcup D^{\prime}, p_{0}\right) .
$$

By Proposition 4.10 the induced homomorphisms

$$
\begin{array}{ll}
i_{*}: \Lambda\left(D \sqcap D^{\prime}, p_{0}\right) \rightarrow \Lambda\left(D, p_{0}\right), & i_{*}^{\prime}: \Lambda\left(D \sqcap D^{\prime}, p_{0}\right) \rightarrow \Lambda\left(D^{\prime}, p_{0}\right), \\
j_{*}: \Lambda\left(D, p_{0}\right) \rightarrow \Lambda\left(D \sqcup D^{\prime}, p_{0}\right), & j_{*}^{\prime}: \Lambda\left(D^{\prime}, p_{0}\right) \rightarrow \Lambda\left(D \sqcup D^{\prime}, p_{0}\right),
\end{array}
$$

are injective, but not surjective if the involved local control sets are different. Clearly one has $j_{*} \circ i_{*}=j_{*}^{\prime} \circ i_{*}^{\prime}$, i.e., the diagram

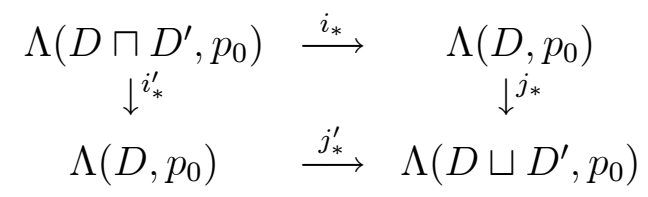

is commutative. 


\section{$5 \quad$ Seifert-Van Kampen theory}

In this section we will study the intersection and the union of two local control sets, assuming that $D \sqcap D^{\prime}$ and $D \sqcup D^{\prime}$ are well defined. However, we cannot describe the relations between the subsemigroups $j_{*}\left(\Lambda\left(D, p_{0}\right)\right)$ and $j_{*}^{\prime}\left(\Lambda\left(D^{\prime}, p_{0}\right)\right)$ of $\Lambda\left(D \sqcup D^{\prime}, p_{0}\right)$ and $\Lambda\left(D \sqcup D^{\prime}, p_{0}\right)$. In the analogous situation for the fundamental group in algebraic topology, one "decomposes" the big loops in $D \cup D^{\prime}$ that cross int $\left(D \cap D^{\prime}\right)$ by inserting paths to the base point $p_{0}$ and their inverses (see, e.g., tom Dieck, Satz 3.7). In our context, however, inverses do not always exist, so this procedure does not apply. In fact, a periodic trajectory in $P\left(D \sqcup D^{\prime}, p_{0}\right)$ with $p_{0} \in \operatorname{int}\left(D \sqcap D^{\prime}\right)$ cannot, in general, be written as the concatenation of periodic trajectories in $P\left(D, p_{0}\right)$ and $P\left(D^{\prime}, p_{0}\right)$. As a consequence, the semigroup $\Lambda\left(D \sqcup D^{\prime}, p_{0}\right)$ need not be generated by the images $j_{*}\left(\Lambda\left(D, p_{0}\right)\right)$ and $j_{*}^{\prime}\left(\Lambda\left(D^{\prime}, p_{0}\right)\right)$. To overcome these difficulties, we will identify $\operatorname{cl}\left(D \sqcap D^{\prime}\right)$ with a point and consider periodic trajectories in the resulting space (actually ignoring the behavior within $D \sqcap$ $D^{\prime}$ ). To make this statement precise, we will construct the notion of relative fundamental semigroup.

For local control sets $D \subset D^{\prime}, p_{0} \in$ int $D$ and $\operatorname{cl} D$ compact we consider the quotient set $D^{\prime} / \mathrm{cl} D$ and let $\pi: D^{\prime} \rightarrow D^{\prime} / \operatorname{cl} D$ be the canonical projection. Let $\mathrm{d}^{\prime}$ be the metric on this space given by

$$
\begin{aligned}
\mathrm{d}^{\prime}(x, y) & =\mathrm{d}(x, y) \text { for } x, y \in D^{\prime} \backslash \operatorname{cl} D, \\
\mathrm{~d}^{\prime}(x, \operatorname{cl} D) & =\min \{\mathrm{d}(x, y), y \in \operatorname{cl} D\} .
\end{aligned}
$$

Note that the quotient topology coincides with the one induced by $\mathrm{d}^{\prime}$. Given $x \in P\left(D^{\prime}, p_{0}\right)$ we denote by $\pi x$ the map $t \mapsto \pi(x(t))$. For technical reasons, we have to restrict our attention to special trajectories: If they hit $\operatorname{cl} D$ then they actually enter $D$. More precisely, we will require the following additional property.

Definition 5.1 A trajectory $x \in P\left(D^{\prime}, p_{0}\right)$ is nontangential to $D$ if

$$
x(t) \in \partial D \text { implies } x(t) \in \partial^{e x} D \cup \partial^{e n} D .
$$

Since by Lemma 2.5 the boundary $\partial D$ is the disjoint union of the exit, entrance and tangential boundaries, it follows that a trajectory $x \in P\left(D^{\prime}, p_{0}\right)$ is nontangential to $D$ iff $x(t) \notin \partial^{t g} D$ for all $t$. Now define

$$
P\left(D^{\prime}, D, p_{0}\right)=\left\{\pi x, x \in P\left(D^{\prime}, p_{0}\right) \text { is nontangential to } D\right\}
$$


endowed with the metric topology given by

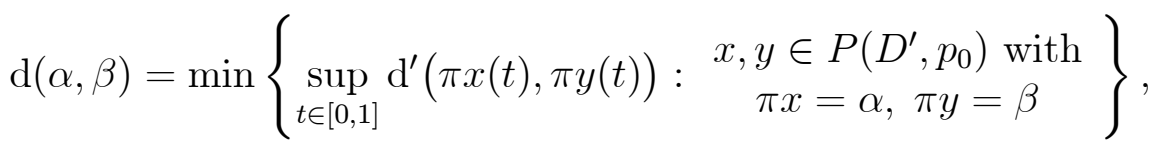

for $\alpha, \beta \in P\left(D^{\prime}, D, p_{0}\right)$. Clearly the distance $\mathrm{d}^{\prime}(x, y)$ depends only on $\alpha$ and $\beta$ (and not on the choice of $x \in \pi^{-1}(\alpha)$ and $y \in \pi^{-1}(\beta)$ ). Thus,

$$
\mathrm{d}(\alpha, \beta)=\sup _{t \in[0,1]} \mathrm{d}^{\prime}(\pi x(t), \pi y(t))
$$

for any $x$ and $y$ such that $\pi x=\alpha$ and $\pi y=\beta$.

We say that $\alpha, \beta \in P\left(D^{\prime}, D, p_{0}\right)$ are homotopic if there exists a continuous map $F:[0,1] \rightarrow P\left(D^{\prime}, D, p_{0}\right)$ with $F(0)=\alpha$ and $F(1)=\beta$. In this case, we write $\alpha \simeq \beta$. In other words trajectories $x, y \in P\left(D^{\prime}, p_{0}\right)$ are homotopic relatively to $D$ if their compositions with the canonical projection $\pi: D^{\prime} \rightarrow$ $D^{\prime} / \mathrm{cl} D$ are homotopic. It is clear that this defines an equivalence relation.

Finally, we put

$$
\Lambda\left(D^{\prime}, D, p_{0}\right)=P\left(D^{\prime}, D, p_{0}\right) / \simeq
$$

and denote with square brackets the equivalence classes. We introduce a semigroup operation in $\Lambda\left(D^{\prime}, D, p_{0}\right)$ defining, for $[\alpha]$ and $[\beta]$ in $\Lambda\left(D^{\prime}, D, p_{0}\right)$,

$$
[\alpha] *[\beta]:=[\pi(x * y)]
$$

where $x, y \in P\left(D^{\prime}, p_{0}\right)$ are such that $\pi x=\alpha$ and $\pi y=\beta$. To see that the operation ' $*$ ' is well defined consider $x, y, x^{\prime}, y^{\prime} \in P\left(D^{\prime}, p_{0}\right)$ with $\pi x \simeq$ $\pi x^{\prime}$ and $\pi y \simeq \pi y^{\prime}$, with homotopies $h_{1}:[0,1] \rightarrow P\left(D^{\prime}, D, p_{0}\right)$ and $h_{2}:$ $[0,1] \rightarrow P\left(D^{\prime}, D, p_{0}\right)$ respectively. It is easily checked that $h_{1} * h_{2}:[0,1] \rightarrow$ $P\left(D^{\prime}, D, p_{0}\right)$, defined as in (9), is continuous, hence it establishes a homotopy $\pi x * \pi y \simeq \pi x^{\prime} * \pi y^{\prime}$. Furthermore this operation is associative. Thus we arrive at the following definition.

Definition 5.2 Let $D \subset D^{\prime}$ be local control sets and assume that $D$ is bounded. Fix $p_{0} \in \operatorname{int} D$. Then fundamental semigroup of $D^{\prime}$ relative to $D$ is $\Lambda\left(D^{\prime}, D, p_{0}\right)$ defined in (13) with the operation '*'.

It is immediate to see that $\Lambda\left(D^{\prime}, D, p_{0}\right)$ contains a unity (represented by any periodic trajectory through $p_{0}$ in $\left.D\right)$. A major difficulty in the analysis of 
the relative fundamental semigroup arises from the fact, that there may exist trajectories which are nontangential, but converge uniformly to trajectories which are tangential.

In order to describe the properties of relative fundamental semigroups we first decompose nontangential trajectories into finitely many pieces inside and outside $\operatorname{cl} D$.

Proposition 5.3 Let $D \subset D^{\prime}$ be local control sets with $p_{0} \in \operatorname{int} D$ and consider a trajectory $x \in P\left(D^{\prime}, p_{0}\right)$ which is nontangential to $D$. Then there are times $t_{0}^{+}=0<t_{1}^{-}<t_{1}^{+}<\ldots<t_{k}^{-}<t_{k}^{+}<t_{k+1}^{-}=1$ such that

$$
x\left|\left[t_{i}^{+}, t_{i+1}^{-}\right] \subset \operatorname{cl} D, \quad x\right|\left[t_{i}^{+}, t_{i+1}^{-}\right] \cap \operatorname{int} D \neq \varnothing \text { and } x \mid\left(t_{i}^{-}, t_{i}^{+}\right) \subset D^{\prime} \backslash \operatorname{cl} D .
$$

Furthermore, $x\left(t_{i}^{+}\right) \in \partial^{e n} D$ and $x\left(t_{i}^{-}\right) \in \partial^{e x} D$ for $i=1, \ldots, k$.

Proof. By Lemma 2.6 a trajectory of the control system can enter and leave $\operatorname{cl} D$ only finitely many times. Invoking Theorem 4.1, we see that this is also true for $x \in P\left(D^{\prime}, p_{0}\right)$. Hence there are only finitely many points

$$
0<t_{1}^{-}<t_{2}^{-}<\ldots<t_{k}^{-}<1
$$

with $x\left(t_{i}^{-}\right) \in \partial^{e x}(D)$ and $x(\tau) \notin \mathrm{cl} D$ for all $t \in\left(t_{i}, t_{i}+\delta\right)$ and some $\delta>0$. Furthermore, one finds only finitely many points

$$
0<t_{1}^{+}<t_{2}^{+}<\ldots<t_{l}^{+}<1
$$

with $x\left(t_{i}^{+}\right) \in \partial^{e n}(D)$ and $x(\tau) \notin \operatorname{cl} D$ for all $\tau \in\left(t_{i}^{+}-\delta, t_{i}^{+}\right)$and some $\delta>0$. Clearly, one has that

$$
x\left|\left[0, t_{1}^{-}\right]=x\right|\left[t_{0}^{+}, t_{1}^{-}\right] \subset \operatorname{cl} D .
$$

and $t_{1}^{+}>t_{1}^{-}$. We also know that

$$
x \mid\left(t_{1}^{-}, t_{1}^{+}\right) \subset D^{\prime} \backslash \mathrm{cl} D,
$$

since the trajectory $x$ can enter $\partial D$ only in the exit boundary (which is impossible from the outside) or in the tangential boundary (which is forbidden by assumption) or the entrance boundary (which yields $x\left(t_{1}^{+}\right)$).

From $x\left(t_{1}^{+}\right)$we can, by Lemma 2.7 , leave the entrance boundary of $D$ only by going into the interior of $D$ or into the tangential boundary of $D$. But by 
assumption the trajectory $x$ does not intersect the tangential boundary of $D$. Hence the trajectory enters the interior of $D$ for some $t>t_{1}^{+}$and $t_{1}^{+}<t_{2}^{-}$.

Now we repeat this construction until $k$. Then it must stop.

The times $t_{i}^{ \pm}$specified by Proposition 5.3 will be called the "transition times". The next lemma shows that the transition times can be shifted by homotopies.

Lemma 5.4 Let $\pi x \in P\left(D^{\prime}, D, p_{0}\right)$ with transition times $t_{i}^{ \pm}, i=1, \ldots, k$. Then there exists a continuous function $F:[0,1] \rightarrow P\left(D^{\prime}, D, p_{0}\right)$ with $F(0)=$ $\pi x$ such that $\pi y=F(1)$ has the transition times

$$
s_{i}^{-}=\frac{2 i-1}{2 k} \text { and } s_{i}^{+}=\frac{i}{k}, i=1, \ldots, k .
$$

Proof. By definition

$$
x \mid\left[t_{i}^{+}, t_{i+1}^{-}\right] \subset \operatorname{cl} D \text { and } x \mid\left(t_{i}^{-}, t_{i}^{+}\right) \subset D^{\prime} \backslash \operatorname{cl} D .
$$

Hence $\pi x(t)=\operatorname{cl} D$ for $t \in\left[t_{i}^{+}, t_{i+1}^{-}\right]$. The following arguments illustrate that one can shift the transition times continuously one by one. Let $i=1$ and suppose first that $t_{1}^{-}>\frac{1}{2 k}$. Define a first homotopy $F_{1}(\lambda)=\pi x^{\lambda}, \lambda \in[0,1]$, as follows. With

$$
t_{1}^{\lambda,-}=(1-\lambda) t_{1}^{-}+\lambda \frac{1}{2 k}
$$

let

$$
\begin{aligned}
x^{\lambda}(t) & =x(t) \text { for } t \in\left[t_{1}^{+}, 1\right] \\
x^{\lambda}(t) & =x\left(\frac{t_{1}^{-}}{t_{1}^{\lambda,-}} t\right) \text { for } t \in\left[0, t_{1}^{\lambda,-}\right], \\
x^{\lambda}(t) & =x\left(t_{1}^{-}+\frac{t_{1}^{+}-t_{1}^{-}}{t_{1}^{+}-t_{1}^{\lambda,-}}\left(t-t_{1}^{\lambda,-}\right)\right) \text { for } t \in\left[t_{1}^{\lambda,-}, t_{1}^{+}\right] .
\end{aligned}
$$

All $x^{\lambda}$ are solutions of $\dot{x}(t) \in\left[\gamma^{-}, \gamma^{+}\right] f(x(t), U), t \in[0,1]$ for some $\gamma^{-}, \gamma^{+}>$ 0 and satisfy $x^{\lambda}(0)=x^{\lambda}(1)=p_{0}$. Clearly, $x^{0}=x$ and $x^{1}$ has first transition times $s_{1}^{-}=\frac{1}{2 k}$ and $s_{1}^{+}=t_{1}^{+}$. The map $\lambda \rightarrow x^{\lambda}$ is continuous in the metric on $P\left(D^{\prime}, p_{0}\right)$ and hence in the metric on $P\left(D^{\prime}, D, p_{0}\right)$ given by (12). An analogous construction can be performed if $t_{1}^{-}<\frac{1}{2 k}$ (if $t_{1}^{+}<\frac{1}{2 k}$, we also have to change the next transition time). Proceeding in this way for all $i$ we find the desired continuous function $F$.

Now we begin to study the behavior of trajectories and their transition points under arbitrary homotopies. 
Lemma 5.5 Let $F:[0,1] \rightarrow P\left(D^{\prime}, D, p_{0}\right)$ be continuous. Then for the elements

$$
\pi x^{\lambda}:=F(\lambda) \in P\left(D^{\prime}, D, p_{0}\right), \lambda \in[0,1],
$$

there is an upper bound for the number of transition times.

Proof. Suppose, contrary to our assertion that there are $\lambda_{n} \in[0,1]$ with an unbounded number of corresponding transition times. We may assume that $\lambda_{n} \rightarrow \gamma \in[0,1]$. Note that by continuity of $F$ the image set $F([0,1]) \subset$ $P\left(D^{\prime}, D, p_{0}\right)$ is compact in the uniform topology, hence we also may assume that $\pi x^{\lambda_{n}}$ converges uniformly to $\pi x^{\gamma}$. Observe that for every $\varepsilon>0$ there are only finitely many intervals $\left[a_{j}, b_{j}\right]$ in $[0,1]$ with $b_{j}<a_{j+1}$ such that for all $j$ there are $s_{j} \in\left[a_{j}, b_{j}\right]$ and $r_{j} \in\left(b_{j}, a_{j+1}\right)$ such that $\mathrm{d}\left(\pi x^{\gamma}\left(s_{j}\right), \operatorname{cl} D\right) \geq \varepsilon$ and $\pi x^{\gamma}\left(r_{j}\right)=\mathrm{cl} D$. The exit and entrance times $t_{i}^{\lambda_{n},-}, t_{i}^{\lambda_{n},+}$ must cluster for $n \rightarrow \infty$ and we denote limit points by $\tau_{i}^{\gamma,-}$ and $\tau_{i}^{\gamma,+}$, respectively. For converging subsequences one finds that

$$
\pi x^{\lambda_{n}}\left(t_{i}^{\lambda_{n},-}\right) \rightarrow \pi x^{\gamma}\left(\tau_{i}^{\gamma,-}\right)=\operatorname{cl} D \text { and } \pi x^{\lambda_{n}}\left(t_{i}^{\lambda_{n},+}\right) \rightarrow \pi x^{\gamma}\left(\tau_{i}^{\gamma,+}\right)=\operatorname{cl} D .
$$

Proposition 5.3 implies that $x^{\lambda_{n}}\left(t_{i}^{\lambda_{n},-}\right) \in \partial^{e x} D, x^{\lambda_{n}}\left(t_{i}^{\lambda_{n},+}\right) \in \partial^{e n} D$ and $x^{\lambda_{n}} \mid\left(t_{i}^{\lambda_{n},-}, t_{i}^{\lambda_{n},+}\right) \subset D^{\prime} \backslash \operatorname{cl} D$. Now suppose that $t_{i}^{\lambda_{n},+}-t_{i}^{\lambda_{n},-} \rightarrow 0$ for $n \rightarrow \infty$. Since $\pi x^{\lambda_{n}}$ converge uniformly to the continuous function $\pi x^{\gamma}$ we find that, for $n$ large enough, $\pi x^{\lambda_{n}} \mid\left[t_{i}^{\lambda_{n},-}, t_{i}^{\lambda_{n},+}\right]$ is contained in an $\varepsilon$-neighborhood of the point $\operatorname{cl} D$ in the space $D^{\prime} / \operatorname{cl} D$. Hence $x^{\lambda_{n}} \mid\left[t_{i}^{\lambda_{n},-}, t_{i}^{\lambda_{n},+}\right]$ is contained in an $\varepsilon$-neighborhood of the subset $\operatorname{cl} D$ in the space $D^{\prime}$. For $n$ large enough this contradicts the local maximality property of $D$ if $\varepsilon$ is chosen small enough that the $\varepsilon$-neighborhood is contained in an isolating neighborhood of $D$. Hence $t_{i}^{\lambda_{n},+}-t_{i}^{\lambda_{n},-}$ remains bounded away from 0 . The same argument shows that $x^{\lambda_{n}} \mid\left[t_{i}^{\lambda_{n},-}, t_{i}^{\lambda_{n},+}\right]$ cannot remain within an isolating neighborhood of $\operatorname{cl} D$. Thus there is $\varepsilon>0$ (not depending on $n$ or $i$ ) such that for all $n$ and all $i$ there are times $s_{i, n} \in\left(t_{i}^{\lambda_{n},-}, t_{i}^{\lambda_{n},+}\right)$ with $\mathrm{d}\left(x^{\lambda_{n}}\left(s_{i, n}\right), \operatorname{cl} D\right) \geq \varepsilon$. Since between any two exit times $t_{i}^{\lambda_{n},-}$ there is an entrance time and between any two entrance times $t_{i}^{\lambda_{n},+}$ there is an exit time, we obtain for $n \rightarrow \infty$ that there are infinitely many cluster points $s_{i}$ of $s_{i, n}$ in intervals $\left[\tau_{i}^{\gamma,-}, \tau_{i}^{\gamma,+}\right]$ satisfying $\mathrm{d}\left(\pi x^{\gamma}\left(s_{i}\right), \operatorname{cl} D\right) \geq \varepsilon$ and $\pi x^{\gamma}\left(\tau_{i}^{\gamma,-}\right), \pi x^{\gamma}\left(\tau_{i}^{\gamma,+}\right)=\operatorname{cl} D$. This is impossible, as shown by the argument above.

We also note the following analogue of Proposition 4.10. 
Lemma 5.6 Let $D \subset D^{\prime} \subset D^{\prime \prime}$ be local control sets with $D$ bounded. Then the inclusion map $i: D^{\prime} \rightarrow D^{\prime \prime}$ induces for $p_{0} \in \operatorname{int} D$ an injective homomorphism

$$
i_{+}: \Lambda\left(D^{\prime}, D, x_{0}\right) \rightarrow \Lambda\left(D^{\prime \prime}, D, p_{0}\right) .
$$

Proof. Denote by $\pi^{\prime}$ and $\pi^{\prime \prime}$ the projections onto $D^{\prime} / \operatorname{cl} D$ and $D^{\prime \prime} / \operatorname{cl} D$ respectively. Since $D^{\prime} / \mathrm{cl} D \subset D^{\prime \prime} / \mathrm{cl} D$, one has that $\pi^{\prime}$ and $\pi^{\prime \prime}$ coincide on $D^{\prime} / \mathrm{cl} D$. Consequently, one has a natural immersion $P\left(D^{\prime}, D, p_{0}\right) \hookrightarrow$ $P\left(D^{\prime \prime}, D, p_{0}\right)$. Define $i_{+}: \Lambda\left(D^{\prime}, D, x_{0}\right) \rightarrow \Lambda\left(D^{\prime \prime}, D, p_{0}\right)$ as the map $\alpha \mapsto \pi^{\prime \prime} x$, where $x \in P\left(D^{\prime}, p_{0}\right)$ is such that $\alpha=\pi^{\prime} x$. To see that $i_{+}$is well defined, take periodic trajectories $x, y \in P\left(D^{\prime}, p_{0}\right)$ such that $\pi^{\prime} x \simeq \pi^{\prime} y$ in $P\left(D^{\prime}, D, p_{0}\right)$. We have to show that $\pi^{\prime \prime} x$ and $\pi^{\prime \prime} y$ yield the same element in $\Lambda\left(D^{\prime \prime}, D, p_{0}\right)$, that is that they are homotopic in $P\left(D^{\prime \prime}, D, p_{0}\right)$. Let $h$ be a homotopy in $P\left(D^{\prime}, D, p_{0}\right)$ between $\pi^{\prime} x$ and $\pi^{\prime} y$. The composition $k$ given by

$$
[0,1] \stackrel{h}{\longrightarrow} P\left(D^{\prime}, D, p_{0}\right) \hookrightarrow P\left(D^{\prime \prime}, D, p_{0}\right) .
$$

is a homotopy between $k(0)=\pi^{\prime \prime} h(0)=\pi x$ and $k(1)=\pi^{\prime \prime} h(1)=\pi^{\prime} y$. As in Lemma 4.9 one can prove that $\pi^{\prime \prime} x \in P\left(D^{\prime \prime}, D, p_{0}\right) \backslash P\left(D^{\prime}, D, p_{0}\right)$ and $y \simeq x$ in $P\left(D^{\prime \prime}, D, p_{0}\right)$ imply $y \in P\left(D^{\prime \prime}, D, p_{0}\right) \backslash P\left(D^{\prime}, D, p_{0}\right)$. Injectivity is then obtained with an argument similar to Proposition 4.10.

In the following we assume that local control sets $D$ and $D^{\prime}$ are given and that there exist local control sets $D \sqcup D^{\prime}$ and $D \sqcap D^{\prime}$ with (6) and (7) and that $D \sqcap D^{\prime}$ is bounded. We apply Lemma 5.6 to the inclusions

$$
D \sqcap D^{\prime} \subset D \subset D \sqcup D^{\prime} \text { and } \quad D \sqcap D^{\prime} \subset D^{\prime} \subset D \sqcup D^{\prime}
$$

and denote the induced maps by

$$
\begin{aligned}
& j_{+}: \Lambda\left(D, D \sqcap D^{\prime}, p_{0}\right) \rightarrow \Lambda\left(D \sqcup D^{\prime}, D \sqcap D^{\prime}, p_{0}\right), \\
& j_{+}^{\prime}: \Lambda\left(D^{\prime}, D \sqcap D^{\prime}, p_{0}\right) \rightarrow \Lambda\left(D \sqcup D^{\prime}, D \sqcap D^{\prime}, p_{0}\right) .
\end{aligned}
$$

Since these maps are injective homomorphisms we identify $\Lambda\left(D, D \sqcap D^{\prime}, p_{0}\right)$ and $\Lambda\left(D^{\prime}, D \sqcap D^{\prime}, p_{0}\right)$ with subsemigroups of $\Lambda\left(D \sqcup D^{\prime}, D \sqcap D^{\prime}, p_{0}\right)$. Our aim in the rest of this chapter is to analyze these semigroups.

The following lemma shows that the pieces of a trajectory outside $D \sqcap D^{\prime}$ are either contained in $D$ or in $D^{\prime}$. 
Lemma 5.7 Let $\pi x \in P\left(D, D \sqcap D^{\prime}, p_{0}\right) \subset P\left(D \sqcup D^{\prime}, D \sqcap D^{\prime}, p_{0}\right)$. Then the decomposition according to Proposition 5.3 applied to the local control sets $D \sqcap D^{\prime} \subset D \sqcup D^{\prime}$ yields for all $i$

$$
x \mid\left(t_{i}^{-}, t_{i}^{+}\right) \subset D \backslash \operatorname{cl}\left(D \sqcap D^{\prime}\right) \text { or } x \mid\left(t_{i}^{-}, t_{i}^{+}\right) \subset D^{\prime} \backslash \operatorname{cl}\left(D \sqcap D^{\prime}\right) .
$$

In the first case $x\left(t_{i}^{-}\right) \in \partial^{e x} D^{\prime}$ and $x\left(t_{i}^{+}\right) \in \partial^{e n} D^{\prime}$ and in the second case $x\left(t_{i}^{-}\right) \in \partial^{e x} D$ and $x\left(t_{i}^{+}\right) \in \partial^{e n} D$.

Proof. By Proposition 5.3 we know that for all $i$

$$
x \mid\left[t_{i}^{+}, t_{i+1}^{-}\right] \subset \operatorname{cl}\left(D \sqcap D^{\prime}\right) \text { and } x \mid\left(t_{i}^{-}, t_{i}^{+}\right) \subset\left(D \sqcup D^{\prime}\right) \backslash \operatorname{cl}\left(D \sqcap D^{\prime}\right) .
$$

Suppose that there is $i$ such that for times $t_{i}^{-}<s, s^{\prime}<t_{i}^{+}$, say with $s<s^{\prime}$,

$$
x(s) \in D \backslash \operatorname{cl}\left(D \sqcap D^{\prime}\right) \text { and } x\left(s^{\prime}\right) \in D^{\prime} \backslash \operatorname{cl}\left(D \sqcap D^{\prime}\right) .
$$

Then there must exist a point $x(\tau) \in \partial^{e x}(D)$ with $s<\tau<s^{\prime}$. Let

$$
\sigma=\max \left\{t \geq \tau, x\left(t^{\prime}\right) \in \operatorname{cl} D \text { for all } t^{\prime} \in[\tau, t]\right\} .
$$

Then $\sigma<s^{\prime}$ and $x(\sigma) \in \partial^{e x}(D)$ such that

$$
x(t) \notin \operatorname{cl} D \text { for all } t \in(\sigma, \sigma+\delta) \text { and some } \delta>0 .
$$

Since $x(t) \in D \sqcup D^{\prime} \subset \operatorname{cl}\left(D \cup D^{\prime}\right)$ for $t \in(\sigma, \sigma+\delta)$ it follows that $x(\sigma) \in$ $\operatorname{cl} D \cap \operatorname{cl} D^{\prime}=\operatorname{cl}\left(D \sqcap D^{\prime}\right)$. This is impossible, since $t_{i}^{-}<\sigma<t_{i}^{+}$.

For the second assertion observe that

$$
x\left(t_{i}^{-}\right) \in \partial^{e x}\left(D \sqcap D^{\prime}\right) \text { and } x\left(t_{i}^{+}\right) \in \partial^{e n}\left(D \sqcap D^{\prime}\right) .
$$

and one has either that for all $s \in\left(t_{i}^{-}, t_{i}^{+}\right)$

$$
x(s) \notin D^{\prime} \text { or } x(s) \notin D .
$$

Consider the first case. Since $\operatorname{cl}\left(D \sqcap D^{\prime}\right)=\operatorname{cl} D \cap \operatorname{cl} D^{\prime}$ it follows that at $x\left(t_{i}^{-}\right)$ the trajectory (coming from $\operatorname{int}\left(D \sqcap D^{\prime}\right) \subset \operatorname{int} D \cap \operatorname{int} D^{\prime}$ ) leaves $\operatorname{cl} D^{\prime}$. This is only possible through $\partial^{e x} D^{\prime}$ and $x\left(t_{i}^{-}\right) \in \partial^{e x} D$ follows. Similarly one argues for $x\left(t_{i}^{+}\right) \in \partial^{e n} D^{\prime}$; and analogously in the second case.

The following theorem which is the main result of this paper may be viewed as an analogue of Seifert-Van Kampen's Theorem for fundamental groups (compare e.g. tom Dieck [13], Satz II.5.7.) It shows that the semigroup for the union of two local control sets $D$ and $D^{\prime}$ is the free product of the semigroups for $D$ and $D^{\prime}$ in the category of semigroups. 
Theorem 5.8 Let $D$ and $D^{\prime}$ be local control sets and assume that there exist local control sets $D \sqcup D^{\prime}$ and $D \sqcap D^{\prime}$ with

$$
D \cup D^{\prime} \subset D \sqcup D^{\prime} \subset \operatorname{cl}\left(D \cup D^{\prime}\right)
$$

and

$$
D \sqcap D^{\prime} \subset D \cap D^{\prime} \text { and } \operatorname{cl}\left(D \sqcap D^{\prime}\right)=\operatorname{cl}\left(D \cap D^{\prime}\right)=\operatorname{cl} D \cap \operatorname{cl} D^{\prime} .
$$

Suppose that $D \sqcap D^{\prime}$ is bounded and fix $p_{0} \in \operatorname{int}\left(D \sqcap D^{\prime}\right)$. Then for every pair of homomorphisms $h: \Lambda\left(D, D \sqcap D^{\prime}, p_{0}\right) \rightarrow H$ and $h^{\prime}: \Lambda\left(D^{\prime}, D \sqcap D^{\prime}, p_{0}\right) \rightarrow H$ into a semigroup $H$ there is a unique homomorphism $\phi: \Lambda\left(D \sqcup D^{\prime}, D \sqcap\right.$ $\left.D^{\prime}, p_{0}\right) \rightarrow H$ with

$$
\phi \circ j=h \text { and } \phi \circ j^{\prime}=h^{\prime} .
$$

Proof. First we discuss uniqueness of $\phi$; it will follow from a decomposition of any trajectory in $\Lambda\left(D \sqcup D^{\prime}, D \sqcap D^{\prime}, p_{0}\right)$ into elements of $\Lambda\left(D, D \sqcap D^{\prime} p_{0}\right)$ and of $\Lambda\left(D^{\prime}, D \sqcap D^{\prime}, p_{0}\right)$. Consider an element $\alpha \in \Lambda\left(D \sqcup D^{\prime}, D \sqcap D^{\prime}, p_{0}\right)$ and let $x \in P\left(D \sqcup D^{\prime}, p_{0}\right)$ be such that $[\pi x]=\alpha$, where $\pi$ denotes the projection $D \sqcup D^{\prime} \rightarrow\left(D \sqcup D^{\prime}\right) / \operatorname{cl}\left(D \sqcap D^{\prime}\right)$.

According to Proposition 5.3 then there are transition times $t_{0}^{+}=0<$ $t_{1}^{-}<t_{1}^{+}<\ldots<t_{k}^{-}<t_{k}^{+}<t_{k+1}^{-}=1$ such that for all $i$

$$
x \mid\left[t_{i}^{+}, t_{i+1}^{-}\right] \subset \operatorname{cl}\left(D \sqcap D^{\prime}\right) \text { and } x \mid\left(t_{i}^{-}, t_{i}^{+}\right) \subset\left(D \sqcup D^{\prime}\right) \backslash \operatorname{cl}\left(D \sqcap D^{\prime}\right) .
$$

Proposition 5.7 implies for all $i$

$$
x \mid\left(t_{i}^{-}, t_{i}^{+}\right) \subset D \backslash \operatorname{cl}\left(D \sqcap D^{\prime}\right) \text { or } x \mid\left(t_{i}^{-}, t_{i}^{+}\right) \subset D^{\prime} \backslash \operatorname{cl}\left(D \sqcap D^{\prime}\right) .
$$

Setting $x_{i}^{ \pm}:=x\left(t_{i}^{ \pm}\right)$one has in the first case $x_{i}^{-} \in \partial^{e x} D^{\prime}$ and $x_{i}^{+} \in \partial^{e n} D^{\prime}$ and in the second case $x_{i}^{-} \in \partial^{e x} D$ and $x_{i}^{+} \in \partial^{e n} D$. We will construct elements of $P\left(D, p_{0}\right)$ and $P\left(D^{\prime}, p_{0}\right)$ such that their concatenations under the projection $\pi$ are homotopic to $\pi x$. Then uniqueness follows.

By Lemma 5.4 we may (without changing the homotopy class) assume that the transition times are $t_{i}^{-}=\frac{2 i-1}{2 k}$ and $t_{i}^{+}=\frac{i}{k}$. It will be convenient to transform $x$ (defined on $[0,1]$ ) into the solution of

$$
\dot{z}(t)=\frac{3}{2 k} f\left(z(t), u\left(\frac{3 t}{2 k}\right)\right), t \in\left[0, \frac{2 k}{3}\right],
$$


which we denote by $\tilde{x}$ (compare Theorem 4.1); it has the transition times $t_{i}^{-}=\frac{2 i-1}{3}$ and $t_{i}^{+}=\frac{2 i}{3}$. We denote the trajectory pieces outside $\operatorname{cl}\left(D \sqcap D^{\prime}\right)$ by $x_{i}=\tilde{x} \mid\left[\frac{2 i-1}{3}, \frac{2 i}{3}\right], i=1, \ldots, k$.

Since $x_{i}^{-}=\tilde{x}\left(\frac{2 i-1}{3}\right) \in \partial^{e x}\left(D \sqcap D^{\prime}\right)$ and $x_{i}^{+}=\tilde{x}\left(\frac{2 i}{3}\right) \in \partial^{e n}\left(D \sqcap D^{\prime}\right) \subset D \sqcap D^{\prime}$, there are trajectories $q_{i}^{-}$and $q_{i}^{+}$in $D \sqcap D^{\prime}$ from $p_{0}$ to $x_{i}^{-}$and from $x_{i}^{+}$to $p_{0}$. More precisely, for $i=1, \ldots, k$ there are $s_{i}^{+}, s_{i}^{-}>0$ and $u_{i}^{+}, u_{i}^{-} \in \mathcal{U}$ such that

$$
\varphi\left(s_{i}^{-}, p_{0}, u_{i}^{-}\right)=x_{i}^{-} \text {and } \varphi\left(s_{i}^{+}, x_{i}^{+}, u_{i}^{+}\right)=p_{0} .
$$

Then the concatenated trajectories $\tilde{y}_{i}=q_{i}^{-} \circ x_{i} \circ q_{i}^{+}$for $i=1, \ldots, k$ start in $p_{0}$ and are closed. By Theorem 4.1 they induce by time transformation elements $y_{i}$ in $P\left(D, p_{0}\right)$ and in $P\left(D^{\prime}, p_{0}\right)$, respectively. We adjust these time transformations such that the pieces where $\tilde{y}_{i}(t)$ coincides with $x_{i}(t)$ are just shifted to $[0,1]$ (the time length is $\frac{1}{3}$ ). Thus only the time intervals $\left[0, s_{i}^{-}\right]$and $\left[s_{i}^{+}, 1\right]$ where the trajectory is in $\operatorname{cl}\left(D \sqcap D^{\prime}\right)$, are adjusted to $\left[0, \frac{1}{3}\right]$ and $\left[\frac{2}{3}, 1\right]$, respectively. Under the projection $\pi$ the $y_{i}$ yield elements $\pi y_{i}$ in $P\left(D, D \sqcap D^{\prime}, p_{0}\right)$ and in $P\left(D^{\prime}, D \sqcap D^{\prime}, p_{0}\right)$, respectively. Now consider the concatenated trajectory

$$
\tilde{y}=\left(q_{1}^{-} \circ x_{1} \circ q_{1}^{+}\right) \circ \ldots \circ\left(q_{i}^{-} \circ x_{i} \circ q_{i}^{+}\right) \circ \ldots \circ\left(q_{k}^{-} \circ x_{k} \circ q_{k}^{+}\right) .
$$

Again by Theorem 4.1 this induces via time transformation an element in $P\left(D \sqcup D^{\prime}, p_{0}\right)$; under the projection $\pi$ it yields an element $\pi y$ in $P(D \sqcup$ $\left.D^{\prime}, D \sqcap D^{\prime}, p_{0}\right)$. Identifying $P\left(D, D \sqcap D^{\prime}, p_{0}\right)$ and $P\left(D^{\prime}, D \sqcap D^{\prime}, p_{0}\right)$ with subsemigroups of $P\left(D \sqcup D^{\prime}, D \sqcap D^{\prime}, p_{0}\right)$ one sees that $\pi y$ is the $*$-product of the elements $\pi y_{i}$. On the other hand, the trajectory $\tilde{y}$ coincides outside of $\operatorname{cl}\left(D \sqcap D^{\prime}\right)$ with the $x_{i}$ and hence with $\tilde{x}$ and the transition times coincide. Thus transforming also $\tilde{y}$ back to a function $y$ defined on $[0,1]$ and applying the projection $\pi$ one finds that the trajectories $\pi x$ and $\pi y$ coincide. We conclude

$$
\begin{aligned}
\pi x & =\pi y=\pi y_{1} * \ldots * \pi y_{k} \quad \text { and } \\
\pi y_{i} & \in P\left(D, D \sqcap D^{\prime}, p_{0}\right) \text { or } \pi y_{i} \in P\left(D^{\prime}, D \sqcap D^{\prime}, p_{0}\right) .
\end{aligned}
$$

This implies uniqueness.

Next we construct the map $\phi$. According to Lemma 5.7 and Lemma 5.6 we can write $\alpha \in \Lambda\left(D \sqcup D^{\prime}, D \sqcap D^{\prime}, x_{0}\right)$ as

$$
\alpha=j_{1}\left(\alpha_{1}\right) \ldots j_{k}\left(\alpha_{k}\right)
$$


with $\alpha_{\nu} \in \Lambda\left(D, D \sqcap D^{\prime}, x_{0}\right), j_{\nu}=j$ or the corresponding dashed objects. Then we define

$$
\phi(\alpha)=\prod h_{\nu}\left(\alpha_{\nu}\right)
$$

where, as above, $h_{\nu}=h$ or $h^{\prime}$. If we can show that this is independent of the representation of $\alpha$, we have defined a homomorphism with the desired properties. Hence we show (for $\beta_{\nu}$ defined analogously)

$$
\prod j_{\nu}\left(\alpha_{\nu}\right)=\prod j_{\nu}\left(\beta_{\nu}\right) \text { implies } \prod h_{\nu}\left(\alpha_{\nu}\right)=\prod h_{\nu}\left(\beta_{\nu}\right) .
$$

For all $\nu$ we write

$$
\alpha_{\nu}=\pi y_{\nu} \text { and } \beta_{\nu}=\pi z_{\nu}
$$

with $y_{\nu}, z_{\nu}$ in $P\left(D, D \sqcap D^{\prime}, p_{0}\right)$ or in $P\left(D^{\prime}, D \sqcap D^{\prime}, p_{0}\right)$. By definition of the relative fundamental semigroup, there is a homotopy $F:[0,1] \rightarrow P(D \sqcup$ $\left.D^{\prime}, D \sqcap D^{\prime}, p_{0}\right)$ from the concatenation of the $\pi y_{\nu}$ to the concatenation of the $\pi z_{\nu}$ and we abbreviate $\pi x^{\lambda}=F(\lambda)$. By Lemma 5.5 there is an upper bound on the number of loops occurring along the homotopy. Hence, by introducing trivial factors (corresponding to trajectories in $D \sqcap D^{\prime}$ ), we may assume that along the homotopy the number of factors is constant. We lump together all consecutive factors in $D$ and in $D^{\prime}$, respectively, and we do not start with a trivial loop.

Claim: Let $\gamma \in[0,1]$. If a loop $x^{\gamma} \mid\left(t_{i}^{\gamma,-}, t_{i}^{\gamma,+}\right) \subset D \backslash \operatorname{cl}\left(D \sqcap D^{\prime}\right)$, then for every $t \in\left(t_{i}^{\gamma,-}, t_{i}^{\gamma,+}\right)$ there is $\varepsilon>0$ such that for every $\lambda$ with $|\lambda-\gamma|<\varepsilon$ it follows that $x^{\lambda}(t) \in D \backslash \operatorname{cl}\left(D \sqcap D^{\prime}\right)$, and hence the loop of $x^{\lambda}$ containing $x^{\lambda}(t)$ is also contained in $D \backslash \operatorname{cl}\left(D \sqcap D^{\prime}\right)$. An analogous statement holds for $D^{\prime}$.

Proof of the Claim. The assumption guarantees that for $t \in\left(t_{i}^{\gamma,-}, t_{i}^{\gamma,+}\right)$ the point $x^{\gamma}(t) \in \operatorname{int} D \backslash \operatorname{cl}\left(D \sqcap D^{\prime}\right)$. Hence the claim follows from the uniform convergence of $\pi x^{\lambda}$ to $\pi x^{\gamma}$ for $\lambda \rightarrow \gamma$.

Now consider the first factor $\alpha_{1}=\pi x_{1}=\pi x_{1}^{0}$ and suppose that $x_{1}$ is contained in $D$. Then for all $\lambda>0$, small enough, it follows that $x_{1}^{\lambda}$ is also contained in $D$. In fact, suppose that there are $\lambda_{n} \rightarrow 0$ with $x_{1}^{\lambda_{n}} \subset D^{\prime}$. Pick $t \in\left(t_{1}^{0,-}, t_{1}^{0,+}\right)$. By the claim, it follows for $n$ large enough that $x^{\lambda_{n}}(t) \in D$ and hence $t_{1}^{\lambda_{n},+}<t_{1}^{0,-}$. Arguing as in the proof of Lemma 5.5, one sees that $x\left(\left[t_{1}^{\lambda_{n},-}, t_{1}^{\lambda_{n},+}\right]\right)$ cannot be contained in an isolating neighborhood of $D$. Hence for some $\varepsilon>0$ and all $n$ one finds points $s_{n} \in\left(t_{1}^{\lambda_{n},-}, t_{1}^{\lambda_{n},+}\right)$ with $\mathrm{d}\left(x^{\lambda_{n}}\left(s_{n}\right), \operatorname{cl} D\right) \geq \varepsilon$. Letting $n$ tend to $\infty$ and considering a convergent subsequence, one finds for $s_{1}^{0}=\lim s_{n} \leq t_{1}^{0,-}$ that $\mathrm{d}\left(\pi x^{0}\left(s_{1}^{0}\right), \operatorname{cl} D\right) \geq \varepsilon$. This 
contradicts the definition of $t_{1}^{0,-}$. Hence we see that

$$
\gamma=\sup \left\{\lambda>0, x_{1}^{\lambda^{\prime}} \subset D \text { for all } \lambda^{\prime} \text { with } \lambda \geq \lambda^{\prime}>0\right\}>0 .
$$

We claim that $x_{1}^{\gamma} \subset D$. Suppose to the contrary that $x_{1}^{\gamma} \subset D^{\prime}$. Then arguing as before, we obtain that for $\lambda<\gamma$, with $\gamma-\lambda$ small enough, also $x_{1}^{\lambda} \subset D^{\prime}$ contradicting the definition of $\gamma$. We conclude that $\gamma=1$ and hence for all $\lambda \in[0,1]$ the first factors are in $D$. Then, invoking Lemma 5.4, we can shift the first transition times $t_{1}^{\lambda, \pm}$ to some fixed value in $(0,1)$. Now we proceed by induction and show that for all $\lambda$ we obtain the same sequence of factors in $D$ and in $D^{\prime}$, respectively, with the same transition times. Restricting the homotopy $F$ we find that all these factors are homotopic in $D$ and in $D^{\prime}$, respectively.

Remark 5.9 Theorem 5.8 shows that

$$
\begin{aligned}
& \{e\}=\Lambda\left(D \sqcap D_{\downarrow^{i_{+}^{\prime}}}^{D^{\prime}}, D \sqcap D^{\prime}, p_{0}\right) \stackrel{i_{+}}{\longrightarrow} \quad \Lambda\left(D, D \sqcap D^{\prime}, p_{0}\right) \\
& \Lambda\left(D, D \sqcap D^{\prime}, p_{0}\right) \quad \stackrel{j_{+*}^{\prime}}{\longrightarrow} \Lambda\left(D \sqcup D^{\prime}, D \sqcap D^{\prime}, p_{0}\right)
\end{aligned}
$$

is a push-out in the category of semigroups. Hence $\Lambda\left(D \sqcup D^{\prime}, D \sqcap D^{\prime}, p_{0}\right)$ is uniquely determined up to isomorphisms.

By Proposition 4.10 we can identify $\Lambda\left(D \sqcap D^{\prime}, x_{0}\right)$ with a subsemigroup of $\Lambda\left(D \sqcup D^{\prime}, x_{0}\right)$, and there is a surjective homomorphism

$$
h: \Lambda\left(D \sqcup D^{\prime}, x_{0}\right) \rightarrow \Lambda\left(D \sqcup D^{\prime}, D \sqcap D^{\prime}, x_{0}\right) .
$$

There always exists a unity, which we denote by $e$, in $\Lambda\left(D \sqcup D^{\prime}, D \sqcap D^{\prime}, x_{0}\right)$ (just take the equivalence class of some periodic trajectory $\alpha_{0}$ in $D \sqcap D^{\prime}$ ). We claim that

$$
\left\{\alpha \in \Lambda\left(D \sqcup D^{\prime}, x_{0}\right), h(\alpha)=e\right\}=\Lambda\left(D \sqcap D^{\prime}, x_{0}\right) .
$$

The inclusion " $\supset$ " is obvious. Conversely, $h(\alpha)=e$ means that the periodic orbits representing $\alpha$ in $\Lambda\left(D \sqcup D^{\prime}, x_{0}\right)$ are homotopic in $\left(D \sqcup D^{\prime}\right) / \operatorname{cl}\left(D \sqcap D^{\prime}\right)$ to the projection of the trajectory $\alpha$. If $\alpha$ is not in $\Lambda\left(D \sqcap D^{\prime}, x_{0}\right)$, then there must exist periodic trajectories through $x_{0}$ which are arbitrarily close to $D \sqcap D^{\prime}$ and not in $D \sqcap D^{\prime}$. This contradicts local maximality of this local control set. Thus we obtain the following result. 
Proposition 5.10 Under the assumptions of Theorem 5.8 the sequence

$$
1 \rightarrow \Lambda\left(D \sqcap D^{\prime}, x_{0}\right) \rightarrow \Lambda\left(D \sqcup D^{\prime}, x_{0}\right) \rightarrow \Lambda\left(D \sqcup D^{\prime}, D \sqcap D^{\prime}, x_{0}\right) \rightarrow 1
$$

is exact, in the sense that the second map is injective; the kernel of the third map equals $\Lambda\left(D \sqcap D^{\prime}, x_{0}\right)$ and it is surjective.

Acknowledgment. This work was supported by the Nonlinear Control Network (TMR Project).

\section{References}

[1] J. Aubin And H. Frankowska, Set-Valued Analysis, Birkhäuser, 1990.

[2] F. Colonius and W. Kliemann, The Dynamics of Control, Birkhäuser, 2000.

[3] F. Colonius and M. Spadini, Local uniqueness of control sets for nonlinear systems, in Stability and Stabilization of Nonlinear Systems, D. Aeyels, F. Lamnabhi-Lagarrigue, and A. van der Schaft, eds., Springer-Verlag, 1999, pp. 115-135.

[4] F. Colonius and M. Spadini, Uniqueness of local control sets, submitted to J. Dyn. Control Sys.

[5] G. HÄCKL And K. SchneIDer, Controllability near Takens-Bogdanov points, J. Dyn. Control Sys., 2 (1996), pp. 583-598.

[6] J. HowIE, An Introduction to Semigroup Theory, Academic Press, 1976.

[7] V. Jurdjevic, Geometric Control Theory, Cambridge University Press, 1997.

[8] J. Lawson, Universal Objects in Lie Semigroup Theory. In Positivity in Lie Theory, J. Hilgert, J.D. Lawson, K.-H. Neeb, E.B. Vinberg, eds., de Gruyter Expositions in Mathematics 20 (1995).

[9] William S. Massey, Algebraic Topology. An Introduction, SpringerVerlag, 1977. 
[10] L.A.B. San Martin And A.J. Santana, The homotopy type of Lie semigroups in semisimple Lie groups. Monatsh. Math., 136(2002), pp. 151-173.

[11] A.V. SARYCHEv, On homotopy properties of trajectories for completely nonholonomic differental systems, Doklady Akad. Nauk SSSR, 314 (1990), pp. 1336-1340. English Translation: Doklady, 42(1991), pp. 674-678.

[12] A.V. Sarychev, On topological properties of trajectory space for nonlinear control systems, in: Analysis of Nonlinear Controlled Dynamical Systems, B. Bride, B. Bonnard, G.P. Gautier, I. Kupka, eds., Birkhäuser 1991, pp. 358-371.

[13] T. том Dieck, Topologie, de Gruyter, 1991. 